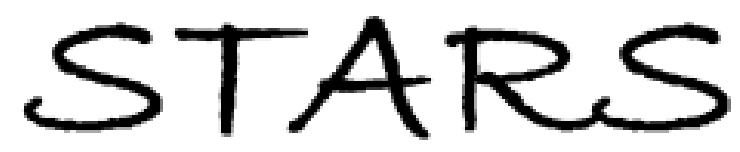

University of Central Florida

STARS

Faculty Bibliography 2000s

Faculty Bibliography

$1-1-2005$

\title{
Fluorescent nanoparticle probes for cancer imaging
}

Swadeshmukul Santra

University of Central Florida

Debamitra Dutta

Glenn A. Walter

Brij M. Moudgil

Find similar works at: https://stars.library.ucf.edu/facultybib2000

University of Central Florida Libraries http://library.ucf.edu

This Review is brought to you for free and open access by the Faculty Bibliography at STARS. It has been accepted for inclusion in Faculty Bibliography 2000 s by an authorized administrator of STARS. For more information, please contactSTARS@ucf.edu.

\section{Recommended Citation}

Santra, Swadeshmukul; Dutta, Debamitra; Walter, Glenn A.; and Moudgil, Brij M., "Fluorescent nanoparticle probes for cancer imaging" (2005). Faculty Bibliography 2000s. 5629.

https://stars.library.ucf.edu/facultybib2000/5629

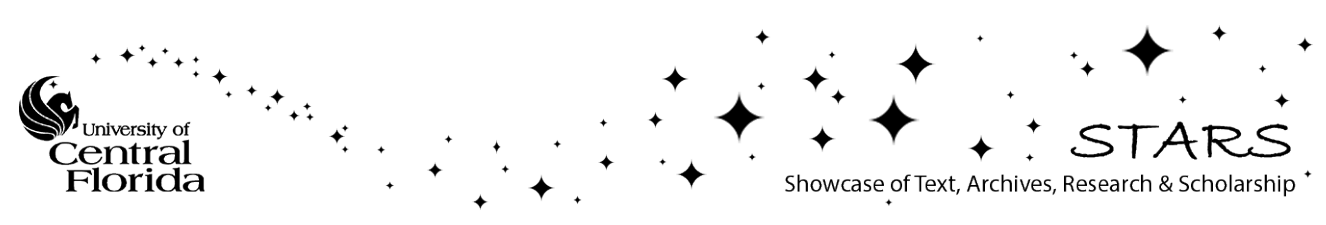




\section{Fluorescent Nanoparticle Probes for Cancer Imaging}

www.tcrt.org

Optical imaging technique has strong potential for sensitive cancer diagnosis, particularly at the early stage of cancer development. This is a sensitive, non-invasive, non-ionizing (clinically safe) and relatively inexpensive technique. Cancer imaging with optical technique however greatly relies upon the use of sensitive and stable optical probes. Unlike the traditional organic fluorescent probes, fluorescent nanoparticle probes such as dye-doped nanoparticles and quantum dots (Qdots) are bright and photostable. Fluorescent nanoparticle probes are shown to be very effective for sensitive cancer imaging with greater success in the cellular level. However, cancer imaging in an in vivo setup has been recently realized. There are several challenges in developing fluorescent nanoparticle probes for in vivo cancer imaging applications. In this review, we will discuss various aspects of nanoparticle design, synthesis, surface functionalization for bioconjugation and cancer cell targeting. A brief overview of in vivo cancer imaging with Qdots will also be presented.

\section{Importance of Diagnostic Cancer Imaging}

According to the American Cancer Society's annual report (1), about 570,280 people are expected to die of various cancers in the year 2005 in the United States of America (USA). Early cancer diagnosis, in combination with the precise cancer therapies could eventually save millions of lives. The diagnosis of cancer at the early stage is extremely challenging and has been an active research area these days. Existing diagnostic non-invasive imaging techniques such as Computed Tomography (CT), Magnetic Resonance (MR), Positron Emission Tomography (PET), Single Photon emission CT (SPECT), and Ultrasound (US) are suitable for the diagnosis of abnormalities in the macroscopic level. However, sensitive imaging of abnormalities in the microscopic level has been very challenging. Substantial research efforts are being made for the development of better cancer imaging techniques in the microscopic level. Several imaging techniques such as, SPECT, PET, and optical techniques have shown great promises $(2,3)$ in the microscopic level. Using fluorescent nanoparticle probes, the feasibility of developing optical imaging technique for the sensitive detection of cancer has been recently demonstrated $(4,5)$.

\section{Nanoparticle Probes for Cancer Tissue Imaging}

Like normal tissues, cancer tissues can interact with light photons by absorption, scattering, and reflection. It is, therefore, expected that the optical image would be somewhat distorted. Again, most tissues autofluoresce (6) upon interaction with the light in the UV and visible spectrum. To develop a robust optical imaging system it is thus important to address all sorts of light interaction with tissue as well as tissue autofluorescence. Again, effective loading of nanoparticle probes to cancer target is important prior to imaging. The concentration of
Swadeshmukul Santra, Ph.D.1,** Debamitra Dutta, M.S.2 Glenn A. Walter, Ph.D. ${ }^{3}$ Brij M. Moudgil, Ph.D.2

${ }^{1}$ Nanoscience Technology Center

Department of Chemistry and

Biomolecular Science Center

University of Central Florida

12424 Research Parkway

Suite 400, Research Pavilion

Orlando, FL 32826, USA

${ }^{2}$ Department of Materials Science and

Engineering and Particle Engineering

Research Center

University of Florida

205 Particle Science and

Technology Building

PO Box 116135

Gainesville, FL 32611, USA

${ }^{3}$ Department of Physiology and

Functional Genomics

University of Florida

PO Box 100274

Gainesville, FL 32610, USA
* Corresponding Author:

Swadeshmukul Santra, Ph.D.

Email: ssantra@mail.ucf.edu 
nanoparticles per unit volume of target tissue would determine the signal strength. Therefore, higher nanoparticle loading is always desirable for better image resolution. Prior to developing nanoparticle probes for cancer tissue imaging the following things should be considered.

Firstly, nanoparticles with the excitation and emission band maxima in the near-infrared (NIR) range $(650 \mathrm{~nm}$ to 900 $\mathrm{nm})$ are highly preferable for deep tissue imaging. The NIR light is capable of penetrating much deeper (up to several centimeters) into the soft tissues and bones $(7,8)$. This is due to the relatively low absorption of tissue components (water and hemoglobin) in the NIR spectral range. Secondly, nanoparticles should have high extinction coefficient for effective absorption and high quantum yield for obtaining strong fluorescence signal. Using such nanoparticles, the sensitivity of the optical imaging could be greatly improved. Fluorescent quantum dots (Qdots) that emit in the visible range have been successfully used in cancer imaging (4, 9-30). However, the excitation and emission of Qdots in the UV range and visible range, respectively, are not desirable and such Qdots will have very limited in vivo applications. To overcome this problem, NIR Qdots have recently been introduced, showing a great promise for in vivo imaging (31-33). The dye-doped nanoparticles (NPs) such as, dye-doped silica (34-37) and dye-doped polymer particles (38-40), present another class of materials for sensitive cancer detection. Thirdly, nanoparticles should be photostable. Photostable nanoparticles will allow non-invasive and real-time monitoring of cancer progression (e.g., monitoring cancer growth and metastasis) and also monitoring the effect of cancer drugs during cancer therapy. Fourthly, nanoparticles should be appropriately surface modified so that they are hydrophilic and an aqueous based formulation can be easily made. Also, they can be attached to appropriate cancer specific delivery systems (e.g., antibodies, peptides, folates, et cetera) for targeting. Lastly, it is also desirable to integrate multiple imaging modalities into a single nanoparticle probe [also called multifunctional nanoparticles (41-50)], making them suitable for cancer imaging using multiple modalities (such as fluorescence, $\mathrm{X}$ ray, MRI, et cetera $(48,51,52))$. This would have great importance for pre-operative cancer diagnosis and intraoperative surgical guidance for cancer tissue resection.

\section{Nanoparticle Probe Development}

Most of the fluorescent nanoparticles (quantum dots, dyedoped silica, et cetera) that have been successfully applied for bioimaging applications such as labeling cancer cells, tissues et cetera, typically possess a core-shell structure (4, 9-30, 34-37) where the core is fluorescent (Figure 1). The nanoparticle shell is to protect the core from photobleaching and to improve nanoparticle dispersibility in aqueous medi- um. The shell structure can also be designed to obtain appropriate surface functional groups for attaching biomolecules. In some cases $(35,48,51,53)$, the core is encapsulated by multiple shell structures. In the following sections a brief overview of the development of Qdots and dyedoped silica nanoparticles are given.

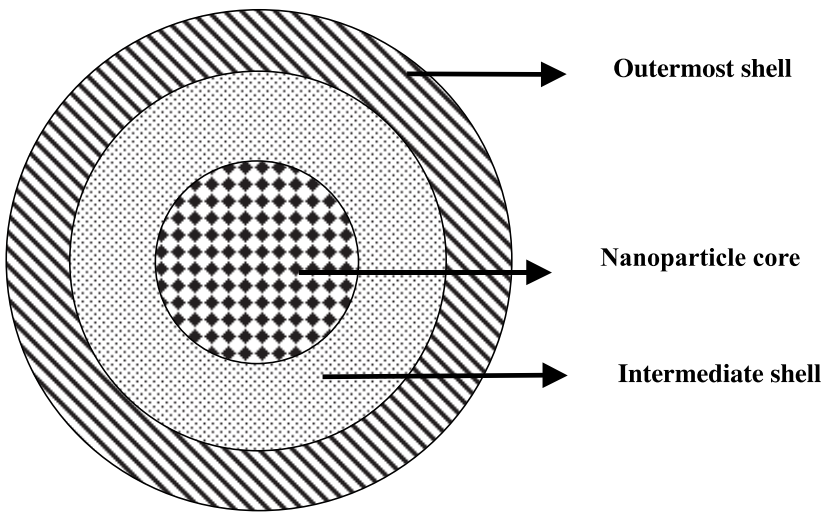

Figure 1: Schematic representation of a typical core-shell nanoparticle design. In some cases, the nanoparticle core is protected just by a single shell (i.e., there is no intermediate shell structure).

\section{Quantum Dots}

Semiconductor Qdots have been extensively used for targeting cancer cells. Qdots are in the size range between 2 and $8 \mathrm{~nm}$. They have broad absorption band with narrow and symmetric emission band (full-width at half-maximum $\sim 25.40 \mathrm{~nm}$ ) and Qdots typically emit in the visible to NIR spectral range $(5,54,55)$. Qdot emission is due to a radiative recombination of an exciton (an electron-hole pair), which is characterized by a long lifetime (56) (>10 ns) leading to the emission of a photon in a narrow and symmetric energy band. In comparison to traditional fluorescent molecules (fluorophores) or fluorescent proteins (e.g., GFP), Qdots have several attractive optical features that are desirable for long-term, multi-target, and highly sensitive bioimaging applications. Some of the major optical features of Qdots are described below.

Firstly, Qdots have large molar extinction coefficient value. Unlike organic fluorescent compounds, Qdots have very large molar extinction coefficient value (57), typically in the order of 0.5-5 × $10^{6} \mathrm{M}^{-1} \mathrm{~cm}^{-1}$ which means that Qdots are capable of absorbing excitation photons very efficiently (the absorption rate is approximately 10-50 times faster than organic dyes). The higher rate of absorption is directly correlated to the Qdot brightness and it has been found that Qdots are approximately 10-20 times brighter than organic dyes (58-60), allowing highly sensitive fluorescence imaging. Secondly, Qdots are extremely photostable. Approximately Qdots are several thousand times more photostable than organic dyes. This feature allows real-time 
monitoring of biological processes over a long period of time. Thirdly, Qdots have much longer lifetime, making them suitable for time-correlated lifetime imaging spectroscopy due to the longer excited state lifetime of Qdots (about one order of magnitude longer than that of organic dyes). This allows effective separation of Qdot fluorescence from the background fluorescence, resulting in improvement in the image contrast by reducing the signal-to-noise ratio dramatically $(61,62)$ in the time-delayed data acquisition mode. Fourthly, Qdots have large Stokes shift value. Unlike in organic dyes, the excitation and the emission spectrum of Qdots are well separated (i.e., large Stokes shift value; typically 300-400 nm depending on the wavelength of the excitation light), allowing further improvement of sensitivity of the detection by reducing the high autofluorescence background often seen in biological specimens (4). Lastly, Qdots have multiple targeting capabilities. The wavelength of Qdot emission is size dependent. The broad Qdot absorption coupled with size dependent emission allows excitation of many sized Qdot particles simultaneously. Unlike organic fluorescent dyes, this unique feature of Qdot materials allows imaging and tracking of multiple targets simultaneously using a single excitation source. This is particularly important in tracking a panel of disease specific molecular biomarkers simultaneously (63). The development of Qdot based fluorescent probes involves a multi-step process, synthesis, surface capping, and bioconjugation. Each of these steps is described below in details.

Synthesis, Surface Modification and Bioconjugation of Qdots: High quality Qdots are highly crystalline, monodispersed (size distribution 8-11\%) and usually synthesized in high boiling point non-polar organic solvents/ligands (mixture of trioctyl phosphine/trioctyl phosphine oxide, TOP/TOPO) at elevated temperature (64). A combination of tri-n-octylphosphine oxide (TOPO) and hexadecylamine has also been reported (55). The mixture serves as a robust reaction medium and also coordinates with unsaturated metal atoms on the Qdot surface to prevent the formation of bulk semiconductors. Following the similar synthesis strategy, Qu et al. (65) has reported the formation high quality CdSe nanocrystals having fluorescence quantum yields as high as $85 \%$ at room temperature. The reverse micelle synthesis route also produce high quality CdS:Mn/ZnS core-shell Qdots (48, 51, 52, 66-68). Reverse micelles [also called water-in-oil (W/O) microemulsion system] is an isotopic, thermodynamically stable homogeneous mixture of oil, water, and surfactant molecules. The surfactant capped water droplets that are dispersed in bulk oil serve as a nano-reactor for the synthesis of Qdots. This method does not require any extreme reaction conditions such as high temperature. Yang et al. $(52,66,67)$ reported the synthesis of manganese doped cadmium sulfide core and zinc sulfide shell (CdS:Mn/ZnS)
Qdots using AOT (dioctylsulfosuccinate sodium salt, a surfactant)/heptane (an oil)/water reverse micelle system. The bright yellow emitting CdS:Mn/ZnS Qdots are small (average Qdot size was $3.2 \mathrm{~nm}$ ) and highly photostable.

The surface passivation of Qdot nanocrystal core are done with a wide bandgap semiconductor materials (shell) such as $\mathrm{ZnS}(58,69)$ to effectively passivate the surface defects $(58,66,70,71)$, protecting the core from photooxidation. As a result of effective surface passivation, Qdot quantum yield increases. According to Derfus et al. (71), adding a shell of 1-2 monolayers of $\mathrm{ZnS}$ produced Qdots less susceptible to oxidation, but did not fully eliminate the cytotoxicity induced by 8 hours of UV photooxidation. The toxicity of Qdots is still an open research question. Surface passivation with silica has been shown to be effective for the CdSe core $(52,72,73)$ nanocrystals. The TOP/TOPOcapped Qdots are hydrophobic and for biological applications, however, it is necessary to obtain aqueous dispersible Qdots. This is usually done by the surface functionalization with hydrophilic ligands. There are three major routes of surface functionalization. Firstly, the "cap exchange" route that involves replacement of TOP/TOPO caping with bifunctional ligands. The bifunctional ligands $(60,74-77)$ have two functional moieties, Qdot surface anchoring (e.g., thiol) and hydrophilic moieties (e.g., hydroxyl, carboxyl). Secondly, the formation of hydrophilic silica shell $(48,51$, $52,59,66,78)$ that encapsulates the Qdot. Lastly, the overcoating of TOP/TOPO-capped Qdots with amphiphilic 'diblock' and 'triblock' copolymers and phospholipids (4, 29, 79-83). Other than these three major surface functionalization routes, Qdot surface has also been capped with mono-mercapto ligands (84), polydentate thiolated ligands (containing more than one thiol groups) $(75,77,84)$ and biocompatible hybrid silica shell $(48,51,52)$. Longer Qdot stability is observed with polydentate thiolated ligands and hybrid silica surface capping.

The bioconjugation represents the attachment of biomolecules (e.g., proteins, antibodies, peptides, DNA, et cetera) to the nanoparticle surface, forming a hybrid structure interfacing both the inorganic and the biological materials, for targeting to biological systems such as cells, tissues, et cetera, either specifically or non-specifically. There are three major avenues to attach proteins to Qdot surface. Firstly, using carbodiimide (e.g., EDC, 1-ethyl-3-(3-dimethylaminopropyl) carbodiimide) coupling chemistry carboxylated Qdots are covalently conjugated to the protein molecules via the formation of stable amide bond. Secondly, peptides can be directly attached to the Qdot surface via the disulfide bond formation between Qdot surface sulfur atoms (from $\mathrm{ZnS}$ surface) and peptides containing cysteine residues $(85,86)$. Histidine expressing proteins (87) or peptides containing polyhistidine residues (88-90) can also be directly attached 
to the $\mathrm{Zn}$ atom on the Qdot surface. Lastly, engineered proteins containing positively charged domains can be noncovalently adsorbed onto the negatively charged Qdot surface via electrostatic interaction $(75,91,92)$.

Cancer Imaging with Quantum Dots: Surface-functionalized quantum dots have been used to image various tumor cells and tissues both in in vitro and in vivo experiments. Some of the different cell lines that have been used are human mammary epithelial tumor (MDA-MB-231) (93), human breast cancer [MDA-MB-435S (94)] [(MDA-MD435 (86) MCF 7 (94) and SK-BR-3 (29)], human prostate cancer (4), squamous carcinoma (95, 96), B16 melanoma (skin cancer) (19), human neuro-blastoma (SK-N-SH) (97), colon tumor (SW480) (94), lung tumor (NCI H1299 (94), and bone tumor (Saos-2) (94) cells. Parak and co-workers used water-soluble (78) siloxane coated quantum dots of two different core sizes $(2.8 \mathrm{~nm}$ with $554 \mathrm{~nm}$ emission and $4.1 \mathrm{~nm}$ with $626 \mathrm{~nm}$ emission) to label human mammary epithelial tumor cells (MDA-MB-231) (93). Confocal microscopic images verified the presence of nanocrystals ingested rapidly inside the cells, and not on the surface. The quantum dot crystals were found in the perinuclear region of the cells even after a week. Akerman and coworkers (86) incubated human breast carcinoma MDA-MD-435 cells with peptide coated quantum dots. These cells were then injected into mice to create tumor grafts and the mice were imaged 8-12 weeks after tumor inoculation. The found that intravenously injected Qdots were accumulated in the MDA-MB-435 breast carcinoma xenograft tumors. Wu et al. (29) used streptavidin conjugated commercial Qdots, QD 560 (emission maximum $560 \mathrm{~nm}$ ), and QD 608 (emission maximum $608 \mathrm{~nm}$ ) to detect Her2 cancer markers on the surface of human breast cancer cells (SK-BR-3). The nanocrystals effectively labeled the cancer cells with negligible affinity to normal cells. Shuming Nie and coworkers (4) have developed multifunctional nanoparticle probes (2.5 $\mathrm{nm}$ radius core protected by a $1 \mathrm{~nm}$ TOPO cap with $2 \mathrm{~nm}$ polymer coating and $5 \mathrm{~nm}$ PEG/affinity ligand shell) for imaging. The quantum dots were used for in vivo imaging to target to tumor sites either through a slow passive targeting process or a more efficient active targeting process. Quantum dots have also been used for in vivo imaging to map sentinel lymph nodes in rats and pigs $(98-100)$. It is known that the presence of lymph node metastases is an early warning signal for breast and lung cancer. Near infrared (NIR) nanocrystals with oligomeric phosphine coating (for solubility in aqueous buffers) was used to guide a surgeon during cancer surgery.

Free cadmium ion is toxic. Qdots can generate free cadmium ions if continuously exposed to excitation source. Most of the current research reports $(54,101)$ have addressed Qdot toxicity in the cellular level and in animal models. It appears that Qdots are safe both in vitro and in vivo if they are appropriately surface coated.

\section{Dye-doped Silica Nanoparticles}

Unlike Qdots, silica does not have inherent strong fluorescence property that can be exploited for sensitive imaging applications. However, silica nanoparticles can be made fluorescent by incorporating fluorescent dye molecules inside the silica matrix (dye-doping). There are several attractive features in silica-based nanoparticles. For example, silica is water dispersible, resistant to microbial attack. The size of silica particles remains unchanged by changing solvent polarity (i.e., resistant to swelling). Silica matrix is optically transparent that allows excitation and emission light to pass through the silica matrix efficiently. Moreover, fluorescent dyes can be effectively entrapped inside the silica particles. The spectral characteristics of the dye molecules remain almost intact. Silica encapsulation provides a protective layer around dye molecules, reducing oxygen molecule penetration (that causes photodegradation of dye molecules) both in air and in aqueous medium (in this case dissolved oxygen). As a result, photostability of dye molecules increases substantially in comparison to bare dyes in solution. However, the photostability of the encapsulated dyes depends on how well dyes are encapsulated by the silica layer (thickness of the silica layer) and whether or not the silica layer is hydrated. In general, better dye stability is observed with thicker and less hydrated silica encapsulation. The dye photostability increased at least by a factor of 100 in the solution state when particles were post-coated with a pure silica shell of about $10 \mathrm{~nm}$ thick. Amorphous silica appears to be a biocompatible (22) and non-toxic (23) material, and have strong potential biological applications. The surface of silica particle can be easily modified to attach biomolecules such as proteins, peptides, antibodies, oligonucleotides, et cetera, using conventional silane based chemistry. For example, carboxylated silica nanoparticles can be covalently attached to the amine groups of proteins, antibodies, et cetera, via the formation of stable amide bond (102). Peptides containing cysteine residue (via, S-H group) can be attached to the aminated silica nanoparticles (51) through (SPDP) coupling chemistry.

\section{Synthesis, Surface Modification and Bioconjugation of} Dye-doped Silica Nanoparticles: A general synthesis strategy of fluorescent silica nanoparticles is the incorporation of organic or metallororganic dye molecules inside the silica matrix (34, 103-109). For example, a metallorganic dye, tris $\left(2,2^{\prime}\right.$-bipyridyl)dichloro ruthenium (II) (Rubpy) has been entrapped inside the silica nanoparticles using a reverse microemulsion based synthesis approach (34) where the positively charged Rubpy molecules were electrostatically bound to the negatively charged silica matrix. The 
dye-doped silica based imaging probes are non-isotopic, sensitive, and relatively photostable in the physiological environment. Additionally, the interaction potential of the silica surface can be easily manipulated to facilitate the interaction with cells (110-112).

There are two synthesis routes reported in the literature for dye doped silica nanoparticle synthesis, Stobers' sol-gel method and reverse microemulsion or water-in-oil (W/O) microemulsion method. In a typical Stobers' method, alkoxysilane compounds (e.g., tetraethyl orthosilicate (TEOS), tetramethylorthosilicate (TMOS), a variety of TEOS, or TMOS derivatives, et cetera) undergo base-catalysed hydrolysis and condensation reaction in ammoniaethanol-water mixture, forming a stable alcosol. This method has been widely used for synthesizing both pure and hybrid (when more than one silane compound are used, such as dye-doped silica particles) silica nanoparticles with particle diameter ranging from a few tens of nanometers to several hundreds of nanometers (sub-micron size). Following the Stobers' protocol with a slight modification, the synthesis of fairly monodisperse organic dye (such as fluorescein isothiocyanate, FITC) doped fluorescent silica nanoparticles has been reported $(35,53)$. The microemulsion method is used for the synthesis of pure silica, as well as inorganic and organic dye-doped silica nanoparticles. The W/O microemulsion is an isotropic, single-phase system that consists of surfactant, oil (as the bulk phase), and water (as nanosize droplets). Each surfactant-coated water droplets that are stabilized in the oil phase serve as an individual nanoreactor for the synthesis of silica nanoparticles. This is a multi-step process that involves the synthesis of dye-doped silica nanoparticle core followed by the formation of pure silica intermediate shell and the outermost shell. The dye-doped nanoparticle core is formed by the hydrolysis and condensation reaction of tetraethylorthosilicate (TEOS) in the presence of ammonium hydroxide $\left(\mathrm{NH}_{4} \mathrm{OH}\right)$ catalyst. Fluorescent dyes are doped during the synthesis of silica core. It is expected that some of the dye molecules will remain of the surface of the core. A pure silica coating (intermediate silica shell) is then applied. The intermediate silica core protects fluorescent dye molecules from photobleaching and also from adverse biological environment that sometimes alters the spectral characteristics of the dye molecules. For bioconjugation, it is necessary to obtain suitable surface functional groups such as carboxyl, thiol, amine, et cetera, on the nanoparticle surface. Also, it is important to consider nanoparticle aqueous dispersibility. A hybrid silica shell (outermost shell) is then formed (53) by the hydrolysis and co-condensation reaction of TEOS, 3aminopropyltriethoxysilane (APTS, an amine containing silane reagent), and (3-trihydroxysilyl)propyl methylphosphonate (THPMP, a negatively charged silane reagent that improves nanoparticle aqueous dispersibility). The fluores- cence brightness of dye-doped silica nanoparticles can be improved by incorporating high-quantum yield organic dyes having large absorption coefficient values. Dye-doped silica nanoparticles in the size range between $30 \mathrm{~nm}$ and 400 $\mathrm{nm}$ have been reported in the literature $(104,113)$.

For bioimaging (e.g., cancer imaging), it is highly desirable that dye-doped silica nanoparticles are appropriately surface modified with cancer targeting molecules such as cancer specific antibodies, folates. This surface modification involves with a few steps. Firstly, the particle surface should be modified to obtain appropriate functional groups such as, amines (35), carboxyls (114), thiols (115), et cetera. Secondly, using suitable coupling reagents, nanoparticles are attached to the bio-recognition molecules (such as antibodies, folates, et cetera). Nanoparticles have also been coated with avidin molecules that are shown to specifically bind biotinalated molecules such as antibodies, proteins, et cetera (116). The surface hydroxyl groups of silica nanoparticles can also be activated by the reactive cyanogens bromide, forming a reactive -OCN derivative. The OCN derivative then readily reacts with proteins (via amine groups), forming a "zerolength" bioconjugate as there is no spacer in between the particles surface and the protein molecule (34). Lastly, bioconjugated particles are targeted to cancers. Note that all these steps are usually carried out in aqueous based solutions.

Cancer Imaging With Dye-doped Silica Nanoparticles: Dye-doped silica nanoparticles have been used for in vitro imaging of cancer cells. Researchers have demonstrated the use of these nanoparticles to label human leukemia $(34,104$, 117), HepG liver cancer (36), human oral carcinoma (35), and lung carcinoma cells $(53,118)$. Tan and coworkers have used $60 \mathrm{~nm}$ dye-doped silica nanoparticles, doped with Rubpy dye to label human leukemia cells $(34,104,117)$. Similarly, He and coworkers (36) have reported a method to recognize HepG liver cancer cells using FITC-APTS doped silica nanoparticles. Recently, our group has developed FITC-doped silica nanoparticles, containing primary amine groups on the particle surface. The FITC-doped silica nanoparticles were then covalently attached to folic acid molecules by a carbodiimide coupling reaction. The affinity of folate immobilized conjugates for folate receptors on the cancer cell surface were utilized for imaging (35). It is known that many cancer cells such as human oral carcinoma (35) and lung carcinoma cells $(53,118$, 119) overexpress folate receptors on the cell surface. Figure 2 showed the effectiveness of folate conjugated nanoparticles for targeting human lung cancer cells. The nanoparticle loaded cells were imaged using a confocal microscope. Control experiments with amine functionalized nanoparticles (without folic acid) and with human dermal fibroblast cells did not result effective cell labeling, suggesting that folate conjugated nanoparticle can be used for labeling A549 cells. This technique could be extended to label other cancer cells. 
Multimodal silica nanoparticle: Multimodal nanoparticles with optical and magnetic properties could help in the preoperative diagnosis and the intraoperative surgical resection of tumors (such as brain tumors, breast cancers et cetera). The synthesis of similar nanoparticles for dual (fluorescence and magnetic) imaging was reported by others $(49,50)$, in which organic fluorescent dyes were used. The use of organic dyes for tumor imaging might be limited as these dyes often cleared rapidly and a large quantity of dyes are needed for optical imaging. Our research group has recently developed single-core multiple-shell novel Rubpy:Gd (III) $/ \mathrm{SiO}_{2}$ nanoparticles that are fluorescent, radio-opaque, and paramagnetic. These nanoparticles might be suitable as a multipurpose imaging probe and can be visualized under CT, MRI, and diffuse optical tomography. The surface reactive groups of this probe can be modified to contain both ligands and antibodies, allowing for the detection of cellular events in vivo.

In order to demonstrate multimodal imaging capability, the following in vitro tests have been performed on Rubpy:Gd (III) $/ \mathrm{SiO}_{2}$ nanoparticles (120). Nanoparticles were first conjugated to folate using carbodiamide coupling chemistry. Upon incubation with human lung cancer cells (A549) for a couple of hours, nanoparticles were internalized by the cancer cells by the receptor-mediated endocytosis process. About one million of nanoparticle loaded A-549 cells were then embedded in $2 \%$ agarose gel. Both the fluorescence and MRI images of the gel were then recorded. Figure 3 clearly showed effective loading of nanoparticles into A-549 cell and multimodal bioimaging capability of these nanoparticles. Moreover, clusters of labeled cells were optically and MR visible.

\section{Gold Nanoparticles and Their Applications for Cancer Imaging}

For more than 30 years, nanometer sized gold particles have primarily been used to stain cells and tissue samples for electron microscopy. However, the use of gold nanoparticles for cancer targeting has been realized very recently (121-123). The basic principle of interactions between gold particles and biomolecules, like proteins, has been well studied for immunocytochemical staining applications. Gold nanoparticles do not fluoresce but effectively scatter light, exhibiting a range of intense colors in the visible and NIR spectral regions. This is known as surface plasmon resonance (124) wrich is caused by the collective oscillation of the conduction electrons induced by the incident electric field (light).

In the solution state, gold nanoparticles are primarily synthesized from gold precursors (e.g., hydrochloroauric acid, $\mathrm{HAuCl}_{4}$ ) using appropriate reducing agents, such as citrate
(125-128), sodium borohydride (129), ascorbic acid (130). Appropriate capping agents are used to control the particle size and size distribution, prevent particle aggregation and stabilize particle solution (such as in aqueous based medium). The citrate reduction of the gold (III) ions has been widely used. While sodium citrate reduces $\left(\mathrm{AuCl}_{4}{ }^{-}\right)$ions in hot aqueous solution, it forms a colloid. The reported average particle size is about $20 \mathrm{~nm}$. Both the citrate ions and its oxidation products (e.g., acetone dicarboxylate) act as capping agents (125-127). Microemulsions (131-133), copolymer micelles (134), reversed micelles (133), surfactant, membranes, and other amphiphiles have also been used for the synthesis of stabilized gold nanoparticles.

Gold bioconjugates have been used for vital imaging of precancerous and cancerous cells by researchers for in vitro and in vivo experiments. The unique optical property of the metal in the nanosized range has been used for detecting breast carcinoma cells (SK-BR-3) (121) breast cancer markers like HER2, oral epithelial live cancer cells (HOC 313 clone 8 and HSC 3) (122), and neoplastic cervical biopsies (123).

\section{Potential of Dye-doped Silica, Quantum Dots, and Gold Nanopaticles}

Quantum dots have been successfully used for imaging cancer cells and tissues. Since Qdots are small $(<10 \mathrm{~nm})$, bright, highly photostable, they might be useful for sensitive real-time monitoring of various intracellular processes in cancer cell. The large Stokes shift value of Qdots is certainly an advantage in reducing the background signal, permitting sensitive detection. The multiplexing capability of Qdots will allow sensitive detection of more than one cancer specific surface receptors at a time using a single excitation. This multiplexing feature might enhance our ability to diagnose cancer at the early stage.

Gold nanoparticles are optically stable. They scatter light strongly and therefore photobleaching does not occur with gold nanoparticles. Unlike Qdots, gold nanoparticles are likely non-toxic. Other than bioimaging, gold nanoparticles can be used for cancer therapy via nanophotothermolysis (135). The size of gold nanoparticles can be varied from a few nanometers to a several tens of nanometers.

Dye doped silica nanoparticles are comparatively larger than Qdots, typically particle size varies from a few tens of nanometers to a couple of hundreds of nanometers. Silica is a biocompatible host matrix for fluorescent dye molecules and it is likely non-toxic in its native form. Dye doped silica are optically more stable than bare dyes. Like Qdots and gold nanoparticles, dye-doped silica nanoparticles are also suitable for diagnostic cancer imaging. 


\section{Conclusions and Perspectives}

Nanoparticle based contrast agents such as quantum dots, gold nanoparticles, dye-doped silica nanoparticles have shown great promise for highly sensitive optical imaging of cancers. As described in this manuscript that most of the successful experiments were conducted in vitro using various cancer cell lines. Only a few animal experiments have been performed so far using quantum dots that demonstrate that quantum dots have strong potential for in vivo cancer imaging. Fluorescent nanoparticle based cancer imaging has strong potential for non-invasive diagnostic cancer imaging at the early stage. However, there are several challenges to overcome for development of highly sensitive and photostable nanoparticles. Nanoparticle surface modification is a critical step for protecting the fluorescent core, improving aqueous dispersibility, and obtaining appropriate surface functionality for bioconjugation. Specific targeting of cancers is important to reduce nono-

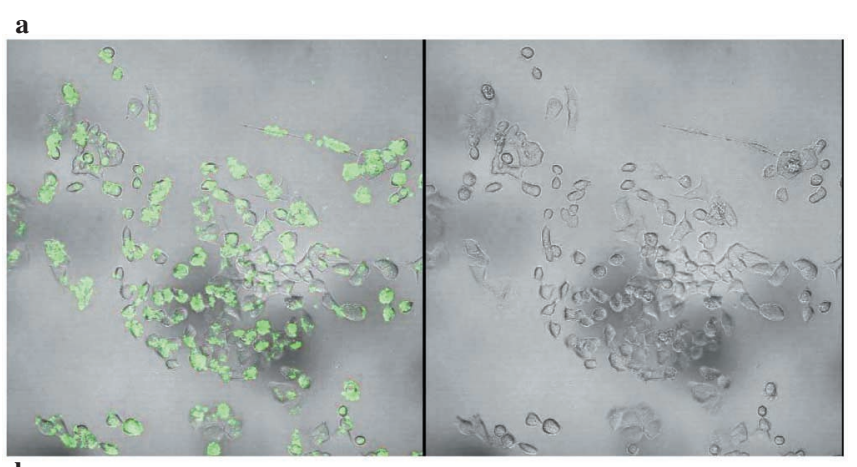
b

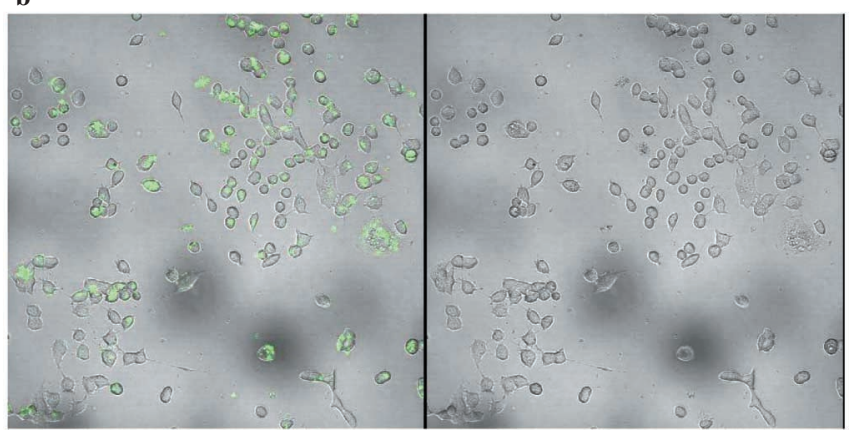

Figure 2: Fluorescence (left) and transmission (right) images of human lung cancer (A549) cells incubated with $200 \mathrm{~nm}$ size folate-conjugated (a) and amine-functionalized (b) fluorescein isothiocyanate (FITC) doped silica nanoparticles. Extra-cellular nanoparticle concentration was $200 \mu \mathrm{g} / \mathrm{ml}$ and the incubation time was 24 hours. This experiment showed more nanoparticle uptake with folate-conjugated nanoparticles, suggesting folate receptor over-expression in A-549 cells. This labeling technique could be extended to label other cancer cells that over-express folate receptors. toxicity related side effects. The use of multifunctional nanoparticles (such as fluorescent and paramagnetic) would have advantage for pre-operative tumor assessment and intra-operative surgical guidance for the tumor tissue resection. Again, the development of nanoparticles with near-infrared excitation and emission would be extremely helpful for deep tissue cancer imaging.

\section{Acknowledgements}

We highly acknowledge Particle Engineering Research Center (PERC), Advanced Magnetic Resonance Imaging and Spectroscopy (AMRIS) and The National High Magnet Field Laboratory at the University of Florida for the support for particle characterization and magnetic measurements.

\section{References}

1. Cancer Facts \& Figures 2005, p. 1. (2005).

2. Sullivan, D. C. Challenges and Opportunities for In Vivo Imaging in Oncology. Technol. Cancer Res. Treat. 1, 419-422 (2002).

3. Lewis, J. S., et al. Small Animal Imaging. Current Technology and Perspectives for Oncological Imaging. Eur. J. Cancer 38, 21732188 (2002).

4. Gao, X. H., et al. In Vivo Cancer Targeting and Imaging with Semiconductor Quantum Dots. Nature Biotechnology 22, 969976 (2004).

5. Medintz, I. L., et al. Quantum Dot Bioconjugates for Imaging, Labelling and Sensing. Nature Materials 4, 435-446 (2005).

6. Bornhop, D. J., et al. Advances in Contrast Agents, Reporters, and Detection. Journal of Biomedical Optics 6, 106-110 (2001).

7. Bremer, C., Ntziachristos, V., and Weissleder, R. Optical-based Molecular Imaging: Contrast Agents and Potential Medical Applications. European Radiology 13, 231-243 (2003).

8. Licha, K. Contrast Agents for Optical Imaging, In Contrast Agents Ii, p. 1-29 (2002)

9. Stroh, M., et al. Quantum Dots Spectrally Distinguish Multiple Species Within the Tumor Milieu In Vivo. Nature Medicine 11, 678682 (2005).

10. Sonvico, F., et al. Metallic Colloid Nanotechnology, Applications in Diagnosis and Therapeutics. Current Pharmaceutical Design 11, 2091-2105 (2005). 
11. Dressler, C., et al. Combination of Bioactive Quantum Dots with an Organic Fluorescent Dye for Two-color Labeling of Cancer Cells Comparison Between Laser-induced and Lamp-induced Fluorescence. Laser Physics 15, 536-544 (2005).

12. Parungo, C. P., et al. Intraoperative Identification of Esophageal Sentinel Lymph Nodes with Near-infrared Fluorescence Imaging. $J$. of Thoracic and Cardiovascular Surgery 129, 844-850 (2005).

13. Ballou, B., Ernst, L. A., and Waggoner, A. S. Fluorescence Imaging of Tumors In Vivo. Current Medicinal Chemistry 12, 795-805 (2005).

14. Ferrari, M. Cancer Nanotechnology: Opportunities and Challenges. Nature Reviews Cancer 5, 161-171 (2005).

15. Lyons, S. K. Advances in Imaging Mouse Tumour Models In Vivo. Journal of Pathology 205, 194-205 (2005).

16. Jiang, W., et al. Semiconductor Quantum Dots as Contrast Agents for Whole Animal Imaging. Trends in Biotechnology 22, 607-609 (2004).

17. Sage, L. Finding Cancer Cells with Quantum Dots. Analytical Chemistry 76, 453A-453A (2004).

18. Chen, G. Y. J. and Yao, S. Q. Lighting up Cancer Cells with "Dots". Lancet 364, 2001-2003 (2004).

19. Voura, E. B., et al. Tracking Metastatic Tumor Cell Extravasation with Quantum Dot Nanocrystals and Fluorescence Emission-scanning Microscopy. Nature Medicine 10, 993-998 (2004).

20. Wu, X. Y., Harper, T. F. and Ng, C. H. Simultaneous Detection of HER2 Gene Amplification and HER2 Protein Expression in Breast Cancer Cells with Semiconductor Nanocrystal Quantum Dots. Faseb Journal 18, C103-C103 (2004).

21. Quantum Dots Target, Image Cancer Cells. Chemical \& Engineering News 82, 40-40 (2004).

22. Minet, O., Dressler, C., and Beuthan, J. Heat Stress Induced Redistribution of Fluorescent Quantum Dots in Breast Tumor Cells. Journal of Fluorescence 14, 241-247 (2004).

23. Beuthan, J., Dressler, C., and Minet, O. Laser-induced Fluorescence Detection of Quantum Dots Redistributed in Thermally Stressed Tumor Cells. Laser Physics 14, 213-219 (2004).

24. Sokolov, K., et al. Optical Systems for In Vivo Molecular Imaging of Cancer. Technology in Cancer Research \& Treatment 2, $491-504$ (2003).

25. Uren, R. F. Cancer Surgery Joins the Dots. Nature Biotechnology 22, 38-39 (2004).

26. Kim, S., et al. Near-infrared Fluorescent Type II Quantum Dots for Sentinel Lymph Node Mapping. Nature Biotechnology 22, 93 97 (2004).

27. Wu, X. Y., et al. Immunofluorescent Labeling of Cancer Marker Her2 and Other Cellular Targets with Semiconductor Quantum Dots (Vol 21, pg 41, 2003). Nature Biotechnology 21, 452-452 (2003).

28. Ben-Ari, E. T. Nanoscale Quantum Dots Hold Promise for Cancer Applications. Journal of the National Cancer Institute 95, 502504 (2003).

29. Wu, X. Y., et al. Immunofluorescent Labeling of Cancer Marker Her2 and Other Cellular Targets with Semiconductor Quantum Dots. Nature Biotechnology 21, 41-46 (2003).

30. LaVan, D. A., Lynn, D. M. and Langer, R. Moving Smaller in Drug Discovery and Delivery. Nature Reviews Drug Discovery 1, 77-84 (2002).

31. Morgan, N. Y., et al. Real Time In Vivo Non-invasive Optical Imaging Using Near-infrared Fluorescent Quantum Dots. Acad Radiol. 12, 313-323 (2005).

32. Soltesz, E. G., et al. Intraoperative Sentinel Lymph Node Mapping of the Lung Using Near-infrared Fluorescent Quantum Dots. Ann. Thorac. Surg. 79, 269-277; Discussion 269-277 (2005).

33. Parungo, C. P., et al. Sentinel Lymph Node Mapping of the Pleural Space. Chest 127, 1799-1804 (2005).

34. Santra, S., et al. Conjugation of Biomolecules with Luminophoredoped Silica Nanoparticles for Photostable Biomarkers. Analytical Chemistry 73, 4988-4993 (2001).
35. Santra, S., et al. Folate Conjugated Fluorescent Silica Nanoparticles for Labeling Neoplastic Cells. Journal of Nanoscience and Nanotechnology 5, 899-904 (2005).

36. He, X. X., et al. A Novel Fluorescent Label Based on Organic Dyedoped Silica Nanoparticles for HepG Liver Cancer Cell Recognition. Journal of Nanoscience and Nanotechnology 4, 585-589 (2004).

37. He, X. X., et al. Photostable Luminescent Nanoparticles as Biological Label for Cell Recognition of System Lupus Erythematosus Patients. Journal of Nanoscience and Nanotechnology 2, 317-320 (2002).

38. Stsiapura, V., et al. Functionalized Nanocrystal-tagged Fluorescent Polymer Beads: Synthesis, Physicochemical Characterization, and Immunolabeling Application. Analytical Biochemistry 334, 257265 (2004).

39. Pan, X. Q., Lee, R. J. and Ratnam, M. Penetration into Solid Tumor Tissue of Fluorescent Latex Microspheres: A Mimic of Liposome Particles. Anticancer Research 24, 3005-3008 (2004).

40. Ueno, H., et al. Experimental Study on Fluorescent Microspheres as a Tracer for Sentinel Node Detection. Anticancer Research 25, 821-825 (2005).

41. Guo, J., et al. Organic-dye-coupted Magnetic Nanoparticles Encaged Inside Thermoresponsive PNIPAM Microcapsutes. Small 1, 737-743 (2005).

42. Veiseh, O., et al. Optical and MRI Multifunctional Nanoprobe for Targeting Gliomas. Nano Letters 5, 1003-1008 (2005).

43. del Campo, A., et al. Multifunctional Magnetite and Silica-magnetite Nanoparticles: Synthesis, Surface Activation and Applications in Life Sciences. Journal of Magnetism and Magnetic Materials 293 , 33-40 (2005).

44. Kopelman, R., et al. Multifunctional Nanoparticle Platforms for In Vivo MRI Enhancement and Photodynamic Therapy of a Rat Brain Cancer. Journal of Magnetism and Magnetic Materials 293, 404410 (2005).

45. Spasova, M., et al. Magnetic and Optical Tunable Microspheres with a Magnetite/Gold Nanoparticle Shell. Journal of Materials Chemistry 15, 2095-2098 (2005).

46. Pellegrino, T., et al. On the Development of Colloidal Nanoparticles Towards Multifunctional Structures and Their Possible Use for Biological Applications. Small 1, 48-63 (2005).

47. Xie, H. Y., et al. Cell-targeting Multifunctional Nanospheres with Both Fluorescence and Magnetism. Small 1, 506-509 (2005).

48. Santra, S., et al. Synthesis of Water-dispersible Fluorescent, Radioopaque, and Paramagnetic CdS:Mn/ZnS Quantum Dots: A Multifunctional Probe for Bioimaging. Journal of the American Chemical Society 127, 1656-1657 (2005).

49. Kircher, M. F., et al. A Multimodal Nanoparticle for Preoperative Magnetic Resonance Imaging and Intraoperative Optical Brain Tumor Delineation. Cancer Research 63, 8122-8125 (2003).

50. Huber, M. M., et al. Fluorescently Detectable Magnetic Resonance Imaging Agents. Bioconjugate Chemistry 9, 242-249 (1998).

51. Santra, S., et al. Rapid and Effective Labeling of Brain Tissue Using TAT-conjugated CdS:Mn/ZnS Quantum Dots. Chemical Communications 25, 3144-3146 (2005).

52. Yang, H. S., Holloway, P. H., and Santra, S. Water-soluble Silicaovercoated CdS:Mn/ZnS Semiconductor Quantum Dots. Journal of Chemical Physics 121, 7421-7426 (2004).

53. Santra, S., et al. TAT Conjugated, FITC Doped Silica Nanoparticles for Bioimaging Applications. Chemical Communications 24, 28102811 (2004).

54. Michalet, X., et al. Quantum Dots for Live Cells, In Vivo Imaging, and Diagnostics. Science 307, 538-544 (2005).

55. Gao, X. H., et al. In Vivo Molecular and Cellular Imaging with Quantum Dots. Current Opinion in Biotechnology 16, 63-72 (2005).

56. Efros, A. L. and Rosen, M. The Electronic Structure of Semiconductor Nanocrystals. Annual Review of Materials Science 30, 475-521 (2000). 
57. Leatherdale, C. A., et al. On the Absorption Cross Section of CdSe Nanocrystal Quantum Dots. Journal of Physical Chemistry B 106, 7619-7622 (2002).

58. Dabbousi, B. O., et al. (CdSe)ZnS Core-shell Quantum Dots: Synthesis and Characterization of a Size Series of Highly Luminescent Nanocrystallites. Journal of Physical Chemistry B 101, 9463-9475 (1997).

59. Bruchez, M., et al. Semiconductor Nanocrystals as Fluorescent Biological Labels. Science 281, 2013-2016 (1998).

60. Chan, W. C. W. and Nie, S. M. Quantum Dot Bioconjugates for Ultrasensitive Nonisotopic Detection. Science 281, 20162018 (1998)

61. Jakobs, S., et al. EGFP and DsRed Expressing Cultures of Escherichia Coli Imaged by Confocal, Two-photon and Fluorescence Lifetime Microscopy. FEBS Letters 479, 131-135 (2000).

62. Pepperkok, R., et al. Simultaneous Detection of Multiple Green Fluorescent Proteins in Live Cells by Fluorescence Lifetime Imaging Microscopy. Current Biology: CB 9, 269-272 (1999).

63. Gao, X. H. and Nie, S. M. Molecular Profiling of Single Cells and Tissue Specimens with Quantum Dots. Trends in Biotechnology 21, 371-373 (2003).

64. Murray, C. B., Norris, D. J., and Bawendi, M. G. Synthesis and Characterization of Nearly Monodisperse Cde $(\mathrm{E}=\mathrm{S}, \mathrm{Se}, \mathrm{Te})$ Semiconductor Nanocrystallites. Journal of the American Chemical Society 115, 8706-8715 (1993).

65. Qu, L. and Peng, X. Control of Photoluminescence Properties of CdSe Nanocrystals in Growth. Journal of the American Chemical Society 124, 2049-2055 (2002).

66. Yang, H., Santra, S., and Holloway, P. H. Synthesis and Application of Mn-Doped II-VI Semiconductor Nanocrystals. Journal of Nanoscience and Nanotechnology 5, 1364-1375 (2005).

67. Yang, H. S., et al. CdS:Mn Nanocrystals Passivated by ZnS: Synthesis and Luminescent Properties. Journal of Chemical Physics 121, 10233-10240 (2004).

68. Yang, H. and Holloway, P. H. Efficient and Photostable ZnSPassivated CdS:Mn Luminescent Nanocrystals. Advanced Functional Materials 14, 152-156 (2004).

69. Hines, M. A. and Guyot-Sionnest, P. Synthesis and Characterization of Strongly Luminescing ZnS-Capped CdSe Nanocrystals. Journal of Physical Chemistry 100, 468-471 (1996).

70. Chen, F. Q. and Gerion, D. Fluorescent CdSe/ZnS Nanocrystal-peptide Conjugates for Long-term, Nontoxic Imaging and Nuclear Targeting in Living Cells. Nano Letters 4, 1827-1832 (2004).

71. Derfus, A. M., Chan, W. C. W. and Bhatia, S. N. Probing the Cytotoxicity of Semiconductor Quantum Dots. Nano Letters 4, 1118 (2004).

72. Selvan, S. T., et al. Formation of Luminescent CdTe-silica Nanoparticles Through an Inverse Microemulsion Technique. Chemistry Letters 33, 434-435 (2004).

73. Selvan, S. T., Tan, T. T., and Ying, J. Y. Robust, Non-cytotoxic, Silica-coated CdSe Quantum Dots with Efficient Photoluminescence. Advanced Materials 17, 1620-1625 (2005).

74. Goldman, E. R., et al. Avidin: A Natural Bridge for Quantum Dotantibody Conjugates. Journal of the American Chemical Society 124, 6378-6382 (2002).

75. Mattoussi, H., et al. Self-assembly of CdSe-ZnS Quantum Dot Bioconjugates Using an Engineered Recombinant Protein. Journal of the American Chemical Society 122, 12142-12150 (2000).

76. Mitchell, G. P., Mirkin, C. A., and Letsinger, R. L. Programmed Assembly of DNA Functionalized Quantum Dots. Journal of the American Chemical Society 121, 8122-8123 (1999).

77. Uyeda, H. T., et al. Synthesis of Compact Multidentate Ligands to Prepare Stable Hydrophilic Quantum Dot Fluorophores. Journal of the American Chemical Society 127, 3870-3878 (2005).
78. Gerion, D., et al. Synthesis and Properties of Biocompatible Watersoluble Silica-coated CdSe/ZnS Semiconductor Quantum Dots. Journal of Physical Chemistry B 105, 8861-8871 (2001).

79. Osaki, F., et al. A Quantum Dot Conjugated Sugar Ball and its Cellular Uptake on the Size Effects of Endocytosis in the Subviral Region. J. of the American Chemical Society 126, 6520-6521 (2004).

80. Pellegrino, T., et al. Hydrophobic Nanocrystals Coated with an Amphiphilic Polymer Shell: A General Route to Water Soluble Nanocrystals. Nano Letters 4, 703-707 (2004).

81. Mattheakis, L. C., et al. Optical Coding of Mammalian Cells Using Semiconductor Quantum Dots. Analytical Biochemistry 327, 200208 (2004).

82. Ballou, B., et al. Noninvasive Imaging of Quantum Dots in Mice. Bioconjugate Chemistry 15, 79-86 (2004).

83. Dubertret, B., et al. In Vivo Imaging of Quantum Dots Encapsulated in Phospholipid Micelles. Science 298, 1759-1762 (2002).

84. Parak, W. J., et al. Biological Applications of Colloidal Nanocrystals. Nanotechnology 14, R15-R27 (2003).

85. Pinaud, F., et al. Bioactivation and Cell Targeting of Semiconductor $\mathrm{CdSe} / \mathrm{ZnS}$ Nanocrystals with Phytochelatin-related Peptides. Journal of the American Chemical Society 126, 6115-6123 (2004).

86. Akerman, M. E., et al. Nanocrystal Targeting In Vivo. Proceedings of the National Academy of Sciences of the United States of America 99, 12617-12621 (2002).

87. Hainfeld, J. F., et al. Ni-NTA-gold Clusters Target His-tagged Proteins. Journal of Structural Biology 127, 185-198 (1999).

88. Slocik, J. M., Moore, J. T., and Wright, D. W. Monoclonal Antibody Recognition of Histidine-rich Peptide Encapsulated Nanoclusters. Nano Letters 2, 169-173 (2002).

89. Medintz, I. L., et al. A Fluorescence Resonance Energy Transferderived Structure of a Quantum Dot-protein Bioconjugate Nanoassembly. Proceedings of the National Academy of Sciences of the United States of America 101, $9612-9617$ (2004).

90. Medintz, I. L., et al. Reversible Modulation of Quantum Dot Photoluminescence Using a Protein-bound Photochromic Fluorescence Resonance Energy Transfer Acceptor. Journal of the American Chemical Society 126, 30-31 (2004).

91. Hanaki, K., et al. Semiconductor Quantum Dot/Albumin Complex is a Long-life and Highly Photostable Endosome Marker. Biochemical and Biophysical Research Communications 302, 496-501 (2003).

92. Goldman, E. R., et al. Conjugation of Luminescent Quantum Dots with Antibodies Using an Engineered Adaptor Protein to Provide New Reagents for Fluoroimmunoassays. Analytical Chemistry 74, 841-847 (2002).

93. Parak, W. J., et al. Cell Motility and Metastatic Potential Studies Based on Quantum Dot Imaging of Phagokinetic Tracks. Advanced Materials 14, 882-885 (2002).

94. Pellegrino, T., et al. Quantum Dot-based Cell Motility Assay. Differentiation 71, 542-548 (2003).

95. Lidke, D. S., et al. Quantum Dot Ligands Provide New Insights into erbB/HER Receptor-mediated Signal Transduction. Nature Biotechnology 22, 198-203 (2004).

96. Morgan, N. Y., et al. Real Time In Vivo Non-invasive Optical Imaging Using Near-infrared Fluorescent Quantum Dots. Academic Radiology 12, 313-323 (2005).

97. Winter, J. O., et al. Recognition Molecule Directed Interfacing Between Semiconductor Quantum Dots and Nerve Cells. Advanced Materials 13, 1673-1677 (2001).

98. Kim, G. J. and Nie, S. Targeted Cancer Nanotherapy. Nanotoday 8 (8 Supplemental 1), 28-33 (2005).

99. Soltesz, E., et al. Intraoperative Sentinel Lymph Node Mapping of the Lung Using Near-infrared Fluorescent Quantum Dots. Annals of Thoracic Surgery 79, 269-277 (2005).

100. Parungo, C. P., et al. Sentinel Lymph Node Mapping of the Pleural Space. Chest 127, 1799-1804 (2005). 
101. Jaiswal, J. K. and Simon, S. M. Potentials and Pitfalls of Fluorescent Quantum Dots for Biological Imaging. Trends in Cell Biology 14, 497-504 (2004).

102. Qhobosheane, M., Zhang, P., and Tan, W. H. Assembly of Silica Nanoparticles for Two-dimensional Nanomaterials. Journal of Nanoscience and Nanotechnology 4, 635-640 (2004).

103. Harma, H., Soukka, T., and Lovgren, T. Europium Nanoparticles and Time-resolved Fluorescence for Ultrasensitive Detection of Prostate-specific Antigen. Clinical Chemistry 47, 561-568 (2001).

104. Santra, S., et al. Development of Novel Dye-doped Silica Nanoparticles for Biomarker Application. Journal of Biomedical Optics 6, 160-166 (2001).

105. Gao, H. F., et al. Preparation of a Novel Polymeric Fluorescent Nanoparticle. Colloid and Polymer Science 280, 653-660 (2002).

106. Makarova, O. V., et al. Adsorption and Encapsulation of Fluorescent Probes in Nanoparticles. Journal of Physical Chemistry B 103, 9080-9084 (1999).

107. Schlupen, J., et al. Sorption Hysteresis of Pyrene on Zeolite. Colloids and Surfaces a-Physicochemical and Engineering Aspects 156, 335-347 (1999).

108. Charreyre, M. T., et al. Fluorescence Energy-Transfer from Fluorescein to Tetramethylrhodamine Covalently Bound to the Surface of Polystyrene Latex-Particles. Langmuir 11, 24232428 (1995).

109. Charreyre, M. T., et al. Adsorption of Rhodamine-60 Onto Polystyrene Latex-Particles with Sulfate Groups at the Surface. Journal of Colloid and Interface Science 170, 374-382 (1995).

110. Liu, X. J. and Tan, W. H. A Fiber-optic Evanescent Wave DNA Biosensor Based on Novel Molecular Beacons. Analytical Chemistry 71, 5054-5059 (1999).

111. Cordek, J., Wang, X. W. and Tan, W. H. Direct Immobilization of Glutamate Dehydrogenase on Optical Fiber Probes for Ultrasensitive Glutamate Detection. Analytical Chemistry 71, 15291533 (1999)

112. Fang, X. H., et al. Designing a Novel Molecular Beacon for Surfaceimmobilized DNA Hybridization Studies. Journal of the American Chemical Society 121, 2921-2922 (1999).

113. Bagwe, R. P., et al. Optimization of Dye-doped Silica Nanoparticles Prepared Using a Reverse Microemulsion Method. Langmuir 20, 8336-8342 (2004).

114. Wang, S. and Low, P. S. Folate-mediated Targeting of Antineoplastic Drags, Imaging Agents, and Nucleic Acids to Cancer Cells. Journal of Controlled Release 53, 39-48 (1998).

115. Tapec, R., Zhao, X. J. J., and Tan, W. H. Development of Organic Dye-doped Silica Nanoparticles for Bioanalysis and Biosensors. Journal of Nanoscience and Nanotechnology 2, 405-409 (2002).

116. Zhao, X. J., et al. A Rapid Bioassay for Single Bacterial Cell Quantitation Using Bioconjugated Nanoparticles. Proceedings of the National Academy of Sciences of the United States of America 101, 15027-15032 (2004).

117. Qhobosheane, M., et al. Biochemically Functionalized Silica Nanoparticles. Analyst 126, 1274-1278 (2001).

118. Santra, S., Dutta, D. and Moudgil, B.M. Functional Dye-doped Silica Nanoparticles for Bioimaging, Diagnostics and Therapeutics. Food and Bioproducts Processing 83, 136-140 (2005).

119. Lee, J. W., et al. Synthesis and Evaluation of Taxol-Folic Acid Conjugates as Targeted Antineoplastics. Bioorganic \& Medicinal Chemistry 10, 2397-2414 (2002).
120. Santra, S., et al. Synthesis and Characterization of Novel Fluorescent, Radio-opaque and Paramagnetic Silica Nanoparticles for Multimodal Bioimaging Applications. Advanced Materials 17, 2165-2169 (2005).

121. Chen, J., et al. Gold Nanocages: Bioconjugation and Their Potential Use as Optical Imaging Contrast Agents. Nano Letters 5, 473477 (2005).

122. El-Sayed, I. H., Huang, X. H., and El-Sayed, M. A. Surface Plasmon Resonance Scattering and Absorption of Anti-EGFR Antibody Conjugated Gold Nanoparticles in Cancer Diagnostics: Applications in Oral Cancer. Nano Letters 5, 829-834 (2005).

123. Sokolov, K., et al. Real-time Vital Optical Imaging of Precancer Using Anti-epidermal Growth Factor Receptor Antibodies Conjugated to Gold Nanoparticles. Cancer Research 63, 19992004 (2003).

124. Santra, S., et al. Luminescent Nanoparticle Probes for Bioimaging. Journal of Nanoscience and Nanotechnology 4, 590-599 (2004).

125. Turkevitch, J., Stevenson, P. C., and Faraday, H. J. Society 11, 55 (1951).

126. Henglein, A. and Giersig, M. Formation of Colloidal Silver Nanoparticles: Capping Action of Citrate. Journal of Physical Chemistry B 103, 9533-9539 (1999).

127. Link, S., Wang, Z. L., and El-Sayed, M. A. Alloy Formation of Goldsilver Nanoparticles and the Dependence of the Plasmon Absorption on their Composition. Journal of Physical Chemistry B 103, 35293533 (1999)

128. Yonezawa, T. and Kunitake, T. Practical Preparation of Anionic Mercapto Ligand-stabilized Gold Nanoparticles and Their Immobilization. Colloids and Surfaces a-Physicochemical and Engineering Aspects 149, 193-199 (1999).

129. Brust, M., et al. Synthesis and Reactions of Functionalized Gold Nanoparticles. Journal of the Chemical Society-Chemical Communications 16, 1655-1656 (1995).

130. Kohler, J. M., Wagner, J. and Albert, J. Formation of Isolated and Clustered Au Nanoparticles in the Presence of Polyelectrolyte Molecules Using a Flow-through Si Chip Reactor. Journal of Materials Chemistry 15, 1924-1930 (2005).

131. Lin, J., Zhou, W. L. and O'Connor, C. J. Formation of Ordered Arrays of Gold Nanoparticles From CTAB Reverse Micelles. Materials Letters 49, 282-286 (2001).

132. Chiang, C. L., Controlled Growth of Gold Nanoparticles in AOT/ C12E4/Isooctane Mixed Reverse Micelles. Journal of Colloid and Interface Science 239, 334-341 (2001).

133. Chen, F. X., Xu, G. Q., and Hor, T. S. A. Preparation and Assembly of Colloidal Gold Nanoparticles in CTAB-stabilized Reverse Microemulsion. Materials Letters 57, 3282-3286 (2003).

134. Sohn, B. H., et al. Directed Self-assembly of Two Kinds of Nanoparticles Utilizing Monolayer Films of Diblock Copolymer Micelles. Journal of the American Chemical Society 125, 63686369 (2003).

135. Zharov, V. P., et al. Synergistic Enhancement of Selective Nanophotothermolysis with Gold Nanoclusters: Potential for Cancer Therapy. Lasers in Surgery and Medicine 37, 219-226 (2005).

Date Received: September 8, 2005

Date Accepted: November 8, 2005 